ÉGYPTE

monde arabe

\section{Égypte/Monde arabe}

30-31 | 1997

Les visions de l'Occident dans le monde arabe

\title{
Les visions de l'Occident dans le monde arabe : Introduction
}

Jean-Noël Ferrié

\section{(2) OpenEdition}

1 Journals

Édition électronique

URL : https://journals.openedition.org/ema/1594

DOI : 10.4000/ema.1594

ISSN : 2090-7273

Éditeur

CEDEJ - Centre d'études et de documentation économiques juridiques et sociales

Édition imprimée

Date de publication : 30 septembre 1997

Pagination : 13-27

ISSN : 1110-5097

Référence électronique

Jean-Noël Ferrié, «Les visions de l'Occident dans le monde arabe : Introduction », Égypte/Monde arabe [En ligne], 30-31 | 1997, mis en ligne le 08 juillet 2008, consulté le 07 juillet 2022. URL : http:// journals.openedition.org/ema/1594; DOI : https://doi.org/10.4000/ema.1594

Ce document a été généré automatiquement le 7 juillet 2022.

Tous droits réservés 


\title{
Les visions de l'Occident dans le monde arabe : Introduction
}

\author{
Jean-Noël Ferrié
}

\section{NOTE DE L'ÉDITEUR}

«Les visions de l'Occident dans le monde arabe » est le titre d'un programme du Cedej financé par la Fondation Giovanni Agnelli, dont Jean-Noël Ferrié a assuré la responsabilité de 1995 à 1997.

1 Il n'y a pas de «visions de l'Occident "mais des stéréotypes sur l'Occident, des références à l'Occident ou des objets occidentaux engagés dans des séquences discontinues de la vie sociale des sociétés dites «arabo-musulmanes ». Ces séquences sont si discontinues, si limitées parfois à de petites choses du monde quotidien, que l'on ne peut envisager d'opérer une synthèse, comme l'on tenterait de reconstruire un puzzle, car les références à l'Occident n'ont tout simplement pas de plan. L'éclatement n'est pas seulement un fait d'observation mais la structure même du phénomène. Il ne saurait donc être question de tenter, dans l'introduction comme dans les articles qui suivent, une impossible synthèse entre des éléments initialement non appareillés; l'introduction se bornera ainsi à indiquer un cadre de saisie et les articles à fournir des cas, et l'on aimerait dire des «brisures ». Pour autant, il ne s'agit pas d'affirmer que les références à l'Occident sont impensables mais seulement qu'elles ne sont pas cumulables.

2 En revanche, il est possible - et c'est l'ambition du présent numéro - de suivre leur biographie sociale (Appadurai, 1986) et de suggérer plus d'un mécanisme (Elster, 1989) ou « ressorts d'ordre » (Livet et Thévenot, 1994, p. 140) permettant de dépeindre, au moins partiellement, la logique de leur utilisation dans des contextes précis. Dina ElKhawaga s'est intéressée à l'un d'eux, l'homologation, dont l'importance toute particulière en fait, peut-être, un dispositif central puisqu'il se rapporte aux conditions générales d'importation et de relocalisation des références occidentales. J'ai tenté, dans 
ma propre contribution, de décrire, à partir de l'Égypte, quatre mécanismes de référence à l'Occident. C'est, sans doute, presque un paradoxe que de consacrer une recherche sur les "visions de l'Occident", d'isoler des mécanismes plutôt que de décrire des contenus, mais les contenus sont, ici, tellement conjoncturels qu'il serait bien imprudent de s'y fier. Je vais rapidement m'attacher à développer cette idée avant de passer au cadre de saisie.

\section{Les mécanismes de la référence à l'occident}

Contrairement à une façon de penser habituelle - mais néanmoins fausse -les sciences sociales ne livrent pas des résultats comme les instituts de sondage livrent des pronostics sur le résultat des élections. Elles n'ont pas pour but de simplifier le réel mais d'en présenter la complexité sous une forme cependant suffisamment réduite et formalisée pour la rendre pensable. Je n'insiste pas sur le fait que le réductionnisme n'est pas exactement une simplification. Les Américains qui sont très préoccupés par les différences culturelles (et les Français qui commencent à l'être) ont développé des recherches sur la question de la communication interculturelle, supposant que les problèmes entre «Noirs» et «Blancs» ou entre «Américains » et «Grecs » dépendaient d'une mauvaise connaissance mutuelle des cultures en présence. Cette idée est à la fois fausse et dangereuse, en ce qu'elle suppose que les acteurs s'opposent sur des contenus collectifs alors qu'ils s'opposent sur des positions individuelles. C'est l'intérêt d'un court et vigoureux article de Jean-Loup Amselle que d'avoir rappelé cette distinction en montrant comment le "nous" est toujours instrumentalisé par les acteurs et, bien souvent, pris en considération par les spécialistes des sciences sociales ; parce qu'il correspond aux postulats holistes de leurs disciplines (Amselle, 1992). En fait, des identités collectives aux représentations collectives et à leur étude, il n'y a qu'un pas. Les représentations collectives sont censées rendre compte, depuis Durkheim, des raisons de l'action collective et de la possibilité même du lien social. Elles possèdent - $d u$ moins pour ceux qui les utilisent - un caractère objectif, indépendant des interactions qui les mettent en cause. Ce postulat n'est pas soutenable, par rapport à l'état actuel des sciences sociales et, tout particulièrement, de la sociologie. De Garfinkel à Boltanski et Thévenot, la sociologie, sans s'en remettre pour autant à une position «mentaliste » faisant procéder les interactions des constructions psychiques du sujet, a montré comment les références étaient engagées dans les actions et en construisaient le cours sans le surdéterminer (Dodier, 1993). En d'autres termes, les références s'éprouvent dans l'action comme elles ne prennent sens que dans le contexte déterminant de leur usage (Wittgenstein, 1953).

4 Ainsi, c'est bien le point de vue pragmatique qui nous permettra de sortir des apories du monde des représentations collectives. Celles-ci n'étant pas agissantes et ne déterminant pas l'action, elles n'ont pas lieu d'être étudiées. Il s'agit, tout simplement, d'un faux objet. Il doit être évident que, si l'approche par les représentations collectives est fausse en général, elle l'est aussi en particulier; si elle est fausse pour tout le monde, elle est fausse aussi pour le monde arabo-musulman, sans qu'il soit besoin d'en faire la démonstration. En faire la démonstration spécifique signifierait alors que l'on considère ce dernier monde comme un monde à part, et il n'est pas besoin d'avoir lu la littérature d'Edward Said et de ses épigones pour savoir que ce n'est pas le cas. Ceci ne procède pas du respect bien-pensant de la culture d'autrui - qui ressortit toujours de 
l'illusion que les représentations collectives existent et fondent des collectifs - mais de l'épistémologie. La sociologie s'applique à tous les phénomènes sociaux où qu'ils aient lieu. Certes, l'on a voulu nous faire accroire qu'il existait une sociologie arabe ou une sociologie islamique seules à même de traiter des sociétés «arabes" ou « musulmanes ", mais le fait même de définir une spécificité (" arabité », « islamite »), à partir d'une altérité dont on veut se détacher (la "sociologie occidentale»), indique bien les limites étroites, sinon les antinomies, de cette perspective. Autant ne plus même en parler.

5 Il nous reste, bien sûr, à examiner les mécanismes eux-mêmes. Par mécanismes, on entend des dispositifs d'ordre (Livet et Thévenot, 1994), c'est-à-dire la description en généralité d'une façon de produire un phénomène social spécifique, en précisant ses ressorts, mais sans recourir à une théorie sociale d'ensemble. Par exemple, si je dis que le fait de se définir comme "musulman » ressortit de la "solidarité sans consensus » (Kertzer, 1988), la « solidarité sans consensus » est un mécanisme. Par cette expression, on entend le fait de reconnaître une référence sans forcément être d'accord sur le contenu et donc sur son propre mode d'engagement vis-à-vis de celle-ci. Il s'agit bien d'un mécanisme, puisque je décris ainsi en généralité un comportement apparemment spécifique, et que je peux utiliser cette description pour penser également en généralité certaines formes de lien social. Un autre mécanisme, davantage lié aux questions d'identité et donc plus topique par rapport à nos préoccupations, est le mécanisme des "typifications", décrit par Berger et Luckman (1989) dans la suite des travaux de Schutz (1987). Par «typifications", on entend le fait de référer les traits d'une personne à des généralités définissant l'un de ses groupes d'appartenance. On ne connaît pas la personne mais on se la représente à partir de la typification de son groupe. Au fur et à mesure qu'on la connaît, les typifications cessent d'être nécessaires pour se la représenter. En ce sens, la typification offre un appui conventionnel pour la cognition. La «typification » est un mécanisme parce que je peux également décrire en généralité une phrase comme "les Occidentaux sont précis mais immoraux». Elle ressortit de la même nécessité cognitive que des phrases comme « les Anglais n'ont pas de cuisine "; elle sert à fournir des repères communs sur des êtres qu'on ne connaît pas vraiment. Ces repères constituent typiquement un savoir conventionnel parce que ceux qui les utilisent peuvent croire qu'ils sont partagés (Livet, 1994).

6 J'en viens maintenant à l'intérêt de décrire les mécanismes. Le premier intérêt est de nous faire comprendre pourquoi les acteurs font une chose, sans entrer dans une explication ou un savoir spécifique. Cette chose peut être décrite en des termes généraux et la description qui en est faite peut être utilisée ailleurs. Le deuxième intérêt découle du premier : la description de mécanismes ôte d'emblée tout caractère ésotérique aux conduites des gens. Elles nous sont plus compréhensibles en ce qu'elles s'inscrivent dans le même monde. En ce sens, les mécanismes constituent un puissant facteur de mise en équivalence. Le troisième intérêt est de nous éviter la stratégie herméneutique qui consiste à tenter de déduire les raisons d'agir du contenu même des typifications. Paul Veyne (1988) nous a, depuis longtemps, mis en garde sur le danger qu'il y a à croire que les représentations sont le programme des pratiques. Les justifications de l'action figurant dans les typifications et, plus largement, dans les actes de langage, ne sont pas nécessairement les raisons d'agir des protagonistes. Je ne suggère pas, ici, une duplicité ou une ignorance des acteurs face à leurs propres motivations, mais seulement qu'elles sont diverses et que leur transformation en argument de justification est soumise à la dynamique des contextes. Si un Marocain 
participe à une manifestation «contre l'Occident " à propos de la guerre du Golfe, il justifiera son geste par la politique des « deux poids, deux mesures » que les Européens et les Américains sont censés pratiquer. Cette raison est sûrement une bonne raison mais - pour reprendre une distinction de Patrick Pharo (1996) - est-ce une raison d'agir? Le même Marocain, dans une conversation privée, reprochera à la France sa politique des visas qui consiste tout simplement à ne plus en accorder. L'injustice de cette fermeture et le caractère effectivement humiliant qu'elle prend, la façon méprisante dont sont traités les gens qui tentent néanmoins d'obtenir un visa n'est-elle pas une bonne raison pour douter du sens de la justice des « Occidentaux »?

7 Mais est-ce pour autant une raison d'agir? Le (toujours) même Marocain, pourra commenter le résultat de la manifestation à laquelle il a participé contre la guerre du Golfe comme une victoire remporté sur Hassan II. C'est aussi très certainement une bonne raison pour manifester, mais est-ce une raison d'agir? Une même conduite peut ainsi renvoyer à une pluralité de justifications selon les contextes et selon les circonstances mais un mécanisme aussi simple que, précisément, « les justifications de l'action ne sont pas forcément les raisons d'agir » permet d'éviter de transformer les justifications en causes, tout en admettant la validité pour l'acteur de l'ensemble des arguments qu'il donne; en revanche, nous demeurons libres de rechercher, parmi ceux-ci, sa raison d'agir.

En fait, l'étude des mécanismes s'accorde à l'esprit de la philosophie analytique - si l'on peut lui appliquer un terme qu'elle aurait peut-être de bonnes raisons de refuser -, qui consiste à séparer les choses et à éprouver leur pertinence propre, refusant toute appréhension holiste des phénomènes. Cette approche est, somme toute, conforme au caractère non cumulable des références à l'Occident, alors que l'étude des mécanismes nous place à chaque fois au cœur des ressorts de l'action.

\section{Le cadre de saisie}

9 Décrire les références à l'Occident comme des positions individuelles, discontinues et conjoncturelles impliquait de sortir de la sociologie holiste des représentations collectives. D'un point de vue plus immédiatement opératoire, cela impliquait d'abandonner l'idée que les identités existent substantiellement, c'est-à-dire de poser au départ qu'il n'y a ni «Occident », ni « Orient », ni «Orient qui juge l'Occident », ni «Occident qui est jugé par l'Orient ». Les identités n'existent pas en elles-mêmes, elles sont des construits conjoncturels, des performances et rien d'autre. La chose est connue chez les anthropologues, depuis l'ouvrage fondamental dirigé par Fredrik Barth qui montrait le caractère interactionnel des jeux identitaires (1969). On construit une identité pour se distinguer d'autrui. En d'autres termes, les frontières entre les groupes sont conjoncturelles, dynamiques et instrumentales. Être «oriental» aujourd'hui ne peut être mis en rapport avec le fait d'être «oriental » il y a trois siècles; la même chose vaut pour être « arabe» ou pour être « musulman ». Ce n'est que depuis peu que les identités s'inventent comme «traditionnelles » ou « immémoriales » (Bayart, 1996) ou que les identités nationales prétendent s'enraciner dans le passé (Anderson, 1991). Tout cela ne remonte guère au-delà du XIX ${ }^{e}$ siècle. S'inventer comme spécifique, dans un processus interactionnel, implique donc de se distinguer d'autrui, c'est-à-dire d'inventer autrui lui-même à la mesure (inverse) de ce que l'on prétend être. C'est ainsi que l'un des pères fondateurs du «Félibrige »- le mouvement identitariste provençal, 
créé au siècle dernier par Frédéric Mistral - a décrit le taureau provençal - qui était la métonymie de l'homme provençal - en lui prêtant systématiquement les caractères inverses du taureau espagnol (Saumade, 1996). Tout processus identitaire fonctionne de la sorte. Certes, il existe des différences d'un lieu à l'autre, mais ces différences substantielles ne deviennent des différences identitaires que dans la mesure où elles sont construites comme telles par les acteurs (Bromberger, 1994). Des différences substantielles peuvent très bien ne jamais être mobilisées dans un construit identitaire, précisément parce qu'elles ne sont pas jugées pertinentes pour mettre en scène l'identité valorisante à laquelle on prétend. L'Occident des sociétés arabo-musulmanes est donc, volontairement, le négatif de l'identité que ces sociétés se reconnaissent. Mais, on conviendra que cette formulation est peut-être un peu holiste, les sociétés ne pouvant se reconnaître une identité dans la mesure où elle ne pensent pas, puisqu'elles ne sont pas des individus dotés de cognition. La croyance aux sujets collectifs a beaucoup fait en faveur de la croyance dans la pertinence des représentations collectives. On peut parfaitement avancer, en revanche, que le contenu des typifications de l'Occident et des Occidentaux est construit à partir de typifications de soi. Quand un Égyptien affirme, par exemple, que les "Occidentaux sont immoraux », il part du principe que l'une des caractéristiques majeures des Égyptiens est leur moralité. Si le portrait de soi laisse apparaître en négatif le portrait de l'autre, le portrait de l'autre laisse apparaître en négatif le portrait de soi (Dupret, à paraître).

Se pose maintenant un problème que j'aimerais éliminer, car il peut jeter le trouble sur la critique des représentations collectives à laquelle je me suis livré. Les typifications peuvent, certes, être engagées dans des interactions entre des individus spécifiés et dans des contextes précis, elles sont néanmoins - semble-t-il -assimilables à des représentations collectives, en ce sens qu'elles n'ont pas un auteur précis et qu'elles sont collectivement partagées. À vrai dire, une telle interprétation me parait fausse à plus d'un titre. D'abord, parce que les typifications ne sont pas unanimement partagées même si elles sont généralement connues par tout le monde; ensuite, parce qu'elles n'impliquent pas une conduite qui leur soit conforme; enfin, parce que les typifications n'impliquent par elles-mêmes qu'un faible engagement quant à la validité de ce qu'elles énoncent. Certes, on peut considérer qu'elles nous disent quelque chose sur quelque chose, mais l'on aurait bien du mal à leur affecter un sens déterminé, intrinsèque en quelque sorte. En effet, si l'on admet qu'une typification possède une histoire sociale et il convient assurément de l'admettre -, celle-ci ne peut être conçue ni comme une histoire linéaire, ni comme une histoire claire. Ce n'est pas une histoire linéaire, parce que la carrière d'une typification n'est pas continue : elle peut avoir un sens à une époque et être expliquée différemment à une autre, être d'un usage rare à un moment et être répandue à un autre. De plus, son histoire n'est pas claire, car elle peut procéder d'une mystification ou d'une fantaisie. Une représentation - lieu commun, typification, stéréotype... - ne tient pas forcément son origine d'un fait réel ou, plus largement, d'une réalité. Elle peut colporter une fiction. Les travaux d'Alain Boureau, sont illustratifs de ces différents points (1996). En ce sens, faire l'histoire sociale d'une représentation s'avère un exercice compliqué dont on ne sait pas toujours où il nous mènera, sauf, peut-être, au constat que les usages s'expliquent toujours par le présent des usagers. De ce point de vue, il est manifeste qu'une assertion comme les "Occidentaux sont immoraux » n'a de sens que contextualisée, parce que l'origine de la typification a peu de chance d'expliquer pourquoi on l'utilise. 
11 Je me suis, par exemple, attaché à montrer que le principal critère distinctif retenu par les étudiants de théologie de l'université d'Al-Azhar pour rendre compte de ce qui sépare les coptes des musulmans est l'absence de moralité des femmes coptes qui seraient, en cela, comparables aux occidentales qu'elles sont censées copier (Ferrié, à paraître). On pourrait supposer que les étudiants ne font que reprendre là un thème plus ancien, stigmatisant les coptes - confondus avec leur bourgeoisie - pour leur proximité avec les communautés étrangères, l'éducation en français de leurs enfants et la scolarité de leurs filles chez les religieuses. Mais il n'en est rien, tout au moins dans le propos des étudiants qui ne font qu'étendre aux coptes une typification généralement utilisée pour faire valoir la supériorité des musulmans sur les "Occidentaux », dans un contexte où il n'est pas facile de trouver des particularités valorisantes. "L'Islam comme civilisation et comme science » de Muhammad Abduh - ainsi que bien d'autres écrits de réformistes - mettait en avant la prétention des musulmans engagés dans la Réforme à posséder à la fois une civilisation et les fondements des sciences, tout en admettant l'avance des Européens dans ce dernier domaine. Il semble difficile de considérer que ce programme ait véritablement abouti. Le cantonnement moraliste du discours sur la supériorité de l'islam n'est peut-être que la conséquence de ce fait. Cependant, on peut soutenir aussi que cette moralité, principalement fondée sur la pudeur sexuelle, est une constante des sociétés musulmanes (Ayubi, 1991) et, dans ce cas, les étudiants se seraient seulement saisis d'une typification ancienne, n'impliquant pas l'échec du réformisme. Une autre explication, tirée de Peter Brown (1995), est que les restrictions en matière sexuelle sont peut-être le seul moyen d'opérer une distinction entre des personnes qui partagent tout le reste, c'est-à-dire qui ne se distinguent en rien par le mode de vie. Or, telle est bien la situation des Égyptiens musulmans face aux Égyptiens coptes : rien ne les distingue sauf la religion. Bien sûr, cette différence est importante mais elle ne peut logiquement être utilisée pour rendre compte de la différence de religion. Nous aurions affaire à une tautologie qui affirmerait que les coptes sont différents des musulmans parce qu'ils sont coptes. En d'autres termes, l'explication d'une différence ne peut s'énoncer dans l'énonciation de la différence. De plus, si rien ne distingue les coptes des musulmans, pourquoi ne sontils pas musulmans? Et peut-on vraiment, en n'étant pas musulman, être comme un musulman? Pourquoi vaut-il mieux alors être musulman si ne pas l'être ne change rien à part la religion, c'est-à-dire si la différence religieuse ne fait pas l'homme meilleur ? Il est clair que l'explication de la différence séparant les coptes des musulmans doit alors se déplacer dans le domaine de la morale sexuelle où il paraît possible de dire enfin en quoi leur religion rend les musulmans meilleurs. Comme on le voit, l'explication fonctionnaliste des typifications n'implique pas l'investigation historique. L'une et l'autre peuvent aisément être séparées et, vraisemblablement, chacune y gagne. Aussi est-il aisé de concevoir que l'histoire ou, plus exactement, le "passé » ne figure pas dans notre cadre de saisie. Tout ce que le passé nous permet en effet de comprendre est la variation d'une typification, le jeu constant des substantiaisations ponctuelles qui la constitue comme objet du présent et non l'origine réelle de l'opposition qu'elle exprime ou du point de vue qu'elle sert à soutenir.

12 L'origine causale des problèmes dont on traite n'est donc pas à rechercher dans la diachronie mais dans la synchronie, c'est-à-dire dans la globalisation. On peut considérer que la globalisation n'est pas plus actuelle que passée, et que les périodes précédentes de l'histoire humaine ont connu d'importants phénomènes de diffusion. De fait, il n'existe pas de sociétés isolées. Cependant, on peut être sensible à l'argument 
de Giddens faisant valoir que les relations entre sociétés atteignent une intensité sans précédent (1994). Cette intensité aboutit jusqu'à un certain point à une imbrication des mondes, à un régime de familiarité de l'autre qui ne doit pas être sous-estimé, quand bien même la revendication identitaire impliquerait-elle, comme le souligne Saâdia Radi, la création de l'altérité. Certes, l'irruption de l'altérité, c'est-à-dire la déclaration de "l'insupportabilité » totale d'une chose inventée comme radicalement autre, la violence en somme, peut sembler nier l'existence d'un régime de familiarité. Pourtant, je crois qu'il nous faut admettre de penser les «happenings" anti-occidentaux ou l'assassinat de prêtres catholiques en Algérie sans recourir à la thèse d'une fracture Identitaire. Il existe, au contraire, de bonnes raisons de penser, que la violence - ce que John Keane nomme « l'incivilité » - est à la fois le produit même de la familiarité et une création endogène des sociétés elles-mêmes (Keane, 1996) qui ne découle pas de leur relation objective à autrui ou de la représentation qu'elles s'en font mais de leur propre constitution. Si l'on veut comprendre la violence (pour autant que celle-ci soit de l'ordre du compréhensible) ou les mouvements sociaux faisant référence à "l'Occident", il convient d'intégrer cette étude dans une étude plus générale de la violence ou des mouvements sociaux et non dans une étude de la typification identitaire. Nous ne trouverons donc pas, dans les articles qui suivent, de mise en relation de la violence et des évocations de «l'Occident». Nous avons, au contraire, insisté sur le régime de la familiarité, non par irénisme (car la familiarité n'est pas exclusive de l'antagonisme), mais par souci de décrire l'aspect de loin le plus important de l'engagement des objets « occidentaux » et des références à « l'Occident » dans la vie quotidienne des sociétés arabo-musulmanes. La rhétorique polémique n'est qu'une forme parmi d'autres de la référence à l'Occident.

\section{L'étonnante présence de « l'occident »}

13 Certes, on ne peut pas ignorer toutefois cette étonnante présence rhétorique de «l'Occident », et mon but n'est certainement pas de la nier. De là vient déjà le crédit que l'on accorde à l'idée de l'existence d'une vision globale et négative de Tailleurs occidental dans les sociétés arabo-musulmanes. Cette étonnante présence est toutefois polarisée sur une unique forme de références à "l'Occident» dont l'unité relève, en fait, de ce que Wittgenstein nommait un « air de famille » : on reconnait des traits sans jamais reconnaître un visage. Il est tentant de penser que cette forme est issue de la période réformiste - qui débute à la fin du premier tiers du XIX ${ }^{\mathrm{e}}$ siècle - et répond, d'abord, au constat d'une relation asymétrique ${ }^{1}$ (mais pas encore coloniale), à l'avantage des premières et au détriment des secondes, entre les sociétés de la rive nord de la Méditerranée et celles de la rive sud. Cette relation asymétrique est inversée, dans l'ordre idéel, par l'affirmation de la supériorité morale de l'islam en même temps que par la reconnaissance de ce qu'il y a de bien et de bon dans les sociétés européennes. Ce thème est récurrent chez les penseurs et les propagandistes de la Réforme $^{2}$ Il est central dans de nombreux ouvrages fondés sur la comparaison entre la réussite des Européens et la situation des "États musulmans », comme l'Essai sur les réformes nécessaires aux États musulmans (Khayr ed-Din, 1987). Il semble qu'au fur et à mesure que le réformisme suit sa dynamique paradoxale, de Muhammad Abduh à Sayyid Qutb (Carré, 1984), le modèle européen (puis occidental) s'inverse et devienne le négatif de ce que devrait être la société selon l'islam, le réformisme s'épurant de la comparaison à l'autre pour se centrer sur la re-constitution de soi ${ }^{3}$, c'est-à-dire sur 
l'élaboration d'une «illusion identitaire» pour reprendre l'heureuse expression de Jean-François Bayart (1996). Cette dynamique est bien présente mais elle ne structure pas -je crois l'avoir montré dans le cours de cette introduction - la relation à «l'Occident ». Il est possible de s'intéresser à elle, mais je pense qu'il importe d'abord de la relativiser car, sinon, on lui ferait assumer - contre toute raison sociologique et historique - la responsabilité totalement imaginaire d'une confrontation entre les civilisations. Il est nécessaire, au contraire, de l'intégrer dans un jeu plus complexe, où la relocalisation aisée des objets occidentaux et des références à l'Occident construit un monde de plus en plus partagé.

14 La première partie s'intéresse aux débuts de cette construction - à l'époque réformiste - et à ses prolongements. Les voyageurs marocains en Occident et au Japon (Roussillon), la traduction d'œuvres marxistes en Égypte (Moghith) et l'introduction du droit français en Égypte (Dupret) sont autant d'occasions de montrer comment Tailleurs occidental est entré naguère dans l'ordre de la familiarité. Il est troublant de constater comment l'identité égyptienne est défendue par référence à une forme d'anthropologie - l'anthropologie physique - souvent considérée comme typique du «colonialisme occidental ", sans que l'auteur de cette défense en tienne compte. Mais ceci suggère que la stigmatisation dépend seulement des circonstances d'utilisation d'une référence et non de son origine. L'origine n'existe qu'avec la polémique comme le crime n'existe qu'avec sa dénonciation (Becker, 1985). Par la suite, d'autres articles reviendront sur ce mécanisme, à travers des objets divers comme la bienfaisance ou les manières de boire. Les aléas des traductions de Marx en Égypte permettent, presque caricaturalement, de rendre compte du mécanisme d'homologation. Par homologation, j'entends le fait d'ajouter ou de retrancher quelque chose à une référence ou à un objet importé pour le rendre publiquement acceptable dans le lieu d'importation (Ferrie, 1996). Paradoxalement, cette forme euphémisée de censure, en rendant plus acceptables les produits "occidentaux", contribue certainement davantage à établir un régime de familiarité qu'à préserver une spécificité.

15 La deuxième partie s'intéresse précisément aux rhétoriques, aux discours. Elle ne leur accorde pas, pour autant, une place déterminante comme le voudrait les théoriciens et les épigones du linguistic turn. Si les paroles sont bien des actes - et des actes sociaux -, elles ne sont pas le programme des conduites: reconnaitre l'un n'est pas admettre l'autre. Elles doivent donc être prises pour ce qu'elles valent. Elles révèlent des constructions du sens, des dilemmes plus ou moins partagés, des lieux communs. À travers les propos sur «l'Occident» des jeunes Marocains et des jeunes Égyptiens (Bennani-Chraïbi, Ossman), des prêcheurs coptes et musulmans du Caire (Radi), des ingénieurs syriens et égyptiens (Hanafi), l'impossibilité de totaliser les rhétoriques devient évidente. Leur valeur réside principalement dans leur valeur d'usage. La référence à la morale des "Orientaux " chez les prêcheurs sert d'abord à se définir comme musulmans ou comme coptes puis comme musulmans et coptes appartenant à la même nation. Elle n'est pas engagée dans une relation avec les "Occidentaux ", dès que celle-ci devient interpersonnelle; elle porte sur des "Occidentaux " génériques, sur des vues de l'esprit. L'Occident est également pris dans un discours plus réflexif où il s'agit de préciser les frontières - et donc les caractéristiques - de l'islamité quand celle-ci est européenne (Farag). Il est aussi engagé dans un étrange jeu de miroirs quand il s'agit de "lui» donner, par fierté nationale, une image de conformité surérogatoire aux critères de «civilisation » qui le caractérisent (Saad). Pourquoi être 
soucieux de son image vis-à-vis de l'Occident? Cette attitude semble contradictoire avec une certaine propension à en donner une image négative. Mais elle peut aussi ne pas l'être et, dans ce cas, critiquer "l'Occident " et vouloir être reconnu par lui signifierait la même chose: avoir douloureusement conscience d'une familiarité inégale.

La troisième et dernière partie est dominée par les usages de références et d'objets " occidentaux ». Il s'agit d'étudier des formes et des dynamiques de relocalisation, de traiter en somme de la manière dont des choses de là-bas entrent dans la routine d'ici. L'ailleurs n'est plus une vue de l'esprit mais un ensemble de pratiques comme le fait de militer pour les droits de l'homme (El-Khawaga), d'user des raffinements de la théorie économique pour justifier "l'économie islamique » (Ireton), de donner aux démunis (Haenni), de meubler un salon (Rachik), de boire (Buisson) ou, par exemple, de se disputer (Ferrie). Relocaliser signifie faire d'une chose étrangère une chose locale. Cette opération implique deux conséquences qui peuvent sembler paradoxales mais qui vont de pair : elle soumet la chose étrangère à une forme de recréation par rapport aux nécessités de son nouveau contexte d'usage, en même temps qu'elle installe ce contexte lui-même dans Tailleurs d'où la chose provient. En effet, ni les références ni les objets ne sont neutres: ils imposent toujours des contraintes de formes comme ils sont toujours liés à des "modes d'emploi » qui se déplacent avec eux. Ceci est encore plus vrai dans un monde d'intense communication visuelle. Bien souvent, les objets sont présents sans être référés à leur origine ou sans y être référés de façon polémique. Cette absence (relative) de polémique dans les usages quotidiens est, certainement, le phénomène le plus «massif» en ce qui concerne l'installation des objets et des références de "l'Occident » dans les sociétés arabo-musulmanes. La disparition, si l'on peut ainsi dire, de la généalogie des objets et des références ou son utilisation ludique est très certainement ce qui prévaut aujourd'hui. Certes, des "entrepreneurs de morales» (Becker, 1985) peuvent toujours tenter de revenir à une définition généalogique des objets et des références, mais l'opération est d'autant plus malaisée que ceux-ci font partie de la vie quotidienne. Le football, par exemple, ne sera jamais stigmatisé comme une invention "occidentale "; tout au plus, procédera-t-on à une forme d'homologation consistant à allonger les shorts des joueurs pour les rendre conformes aux normes de la bienséance. La leçon des droits de l'homme est encore plus intéressante de ce point de vue. Quelles que soient les polémiques sur leur origine, ils sont clairement utilisés comme ressources politiques par les acteurs, les dénonciations d'être "à la solde de l'étranger» (de "l'Occident») devenant même un mode de stigmatisation interne aux associations des droits de l'homme. Ceci en dit long sur l'importance de la relocalisation.

17 Au total, rien ne permet d'accréditer l'idée que «l'Occident » serait une chose plutôt qu'une autre. Les références à " l'Occident » sont, au contraire, étroitement liées aux contextes locaux dans lesquels elles sont utilisées et les logiques généalogiques étroitement insérées dans les visées polémiques. Il est tentant alors de considérer que «l'Occident» n'existe pas en dehors de la multitude des actes de langages qui révoquent. Peut-être est-il ce référent vide évoqué par Lévi-Strauss pour rendre compte de la Mana (1983): il rend possible des opérations sans posséder de valeur propre. 


\section{BIBLIOGRAPHIE}

AMSELLE J.-L, 1992, « Quelques réflexions sur la question des identités collectives en France aujourd'hui ", dans FERRIÉ J.-N., BOËTSCH G., Anthropologie de l'immigration, Aix-en-Provence, IREMAM, (Cahiers de IREMAM n²).

ANDERSON B., 1991, Imagined Communities. Reflections on the Origin and Spread of Nationalism, Londres, Verso (édition révisée).

APPADURAI A. (ed.), 1986, The Social Life of Things: Commodities in Cultural Perspectives, Cambridge, Cambridge University Press.

AYUBI N., 1991, "The politics of sex and the family, or the "collectivity" of islamic morality ", inAYUBI N. (ed.), Political Islam. Religion and Politics in the Arab World, Londres, Routledge.

BARTH F, 1969, Ethnic Groups and Boundaries, Boston, Little, Brown and Co.

BAYART J.-F, 1996, L'Illusion identitaire, Paris, Fayard.

BECKER H., 1985, Outsiders, Paris, Métailié.

BERGER P., LUCKMANN I, 1989, La Construction sociale de la réalité, Paris, Méridiens-Klincksieck.

BOUREAU A., 1996, « La compétence inductive. Un modèle d'analyse des représentations rares », dans LEPETIT B., Les Formes de l'expérience. Une autre histoire sociale, Paris, Albin Michel.

BROMBERGER C, 1994, «L'ethnologie de la France et le problème de l'identité », Civilisation, vol. XLII, $n^{\circ} 2$.

BROWN P., 1995, Le Renoncement à la chair. Virginité, célibat et continence dans le christianisme primitif, Paris, Gallimard.

CARRÉO., 1984, Mystique et politique. Lecture révolutionnaire du Coran par Sayyid Qutb, Frère musulman radical, Paris, Presses de la FNSP et Éditions du Cerf.

DODIER N., 1993, « Les appuis conventionnels de l'action. Éléments de pragmatique sociologique ", Réseaux, $\mathrm{n}^{\circ} 62$.

DUPRET B., à paraître, «La définition juridique des appartenances. La typification narrative de l'action identitaire devant les juridictions suprêmes d'Égypte et d'Israël », Journal International de Sémiotique Juridique.

ELSTER J., 1989, Nuts and Bolts for the Social Sciences, Cambridge, Cambridge University Press.

FERRIE J.-N. :

-1996, «L'appartenance des objets. Problèmes d'anthropologie de la culture et de l'identité », Égypte/Monde arabe, $\mathrm{n}^{\circ} 25$.

- à paraître, «Qu'est-ce qu'un copte selon les musulmans ? Construction de la frontière et typification de soi », dans DECOBERT C, Dynamiques identitaires en Égypte.

GIDDENS A., 1994, Conséquences de la modernité, Paris, L'Harmattan.

ISSAWI C, 1982, An Economie History of the Middle East and North Africa, New York, Columbia University Press.

KEANE J., 1996, Reflections on Violence, Londres, Verso.

KERTZER D., 1988, Rituals, Politics and Power, New Haven, Yale University Press. 
KHAYR ED-DIN, 1987, Essai sur les réformes nécessaires aux États musulmans (présentation et annotation, Magali MORSY), Aix-en-Provence, Edisud.

LÉVI-STRAUSS C, 1983, «Introduction », dans MAUSS M., Sociologie et Anthropologie, Paris, PUF.

LIVET P., 1994, La Communauté virtuelle. Action et communication, Combas, L'Éclat.

LIVET P., THÉVENOT L, 1994, « Les catégories de l'action collective », dans ORLÉAN A., Analyse économique des conventions, Paris, PUF.

PHARO P., 1996, L'Injustice et le Mal, Paris, L'Harmattan.

POLK W. R., CHAMBERS R. L., (eds), 1968, Beginnings of Modernization in the Middle East, Chicago, Chicago University Press.

ROUSSILLON A., 1995, Entre réforme sociale et mouvement national : identité et modernisation en Égypte (1882-1962), Le Caire, Cedej.

SAUMADE F., 1996, « Race régionale, identité nationale. Pour une ethnologie des comportements électoraux », Terrains, $n^{\circ} 27$.

SCHUTZ A., 1987, Le Chercheur et le quotidien, Paris, Méridiens-Klincksieck.

VEYNE P., 1988, « Conduites sans croyances et œuvres d'art sans spectateurs », Diogène, n¹43.

WITTGENSTEIN L., 1953, Philosophical Investigations, Oxford, Blackwell.

\section{NOTES}

1. Il s'agit d'une extension de la notion de "asymetrical développement ", proposée par Ch. Issawi (1982). Voir également Polk et Chambers (1968).

2. Il est typique aussi chez les auteurs mineurs, comme le souligne l'article d'Alain Roussillon (1995).

3. Voir l'introduction d'Alain Roussillon (1995).

\section{INDEX}

Mots-clés : visions de l'Occident

\section{AUTEUR \\ JEAN-NOËL FERRIÉ}

Cedej 